\title{
GGAA Microsatellite Repeat
}

National Cancer Institute

\section{Source}

National Cancer Institute. GGAA Microsatellite Repeat. NCI Thesaurus. Code C157581.

A microsatellite containing 18-26 repeats of the tetranucleotide sequence, GGAA.

EWSR1/FLI1 and EWSR1/ETS family fusion proteins bind to GGAA microsatellite sequences in certain gene promoters, which aberrantly upregulates transcription of those genes and is associated with Ewing Sarcoma. 